\title{
Pure SU(3) Topological Susceptibility at Finite Temperature with the Wilson Flow
}

\author{
Szabolcs Borsányi, ${ }^{a}$ Zoltán Fodor, ${ }^{a b c}$ Sándor Katz, ${ }^{c d}$ Simon Mages, ${ }^{* b e}$ Dániel \\ Nógrádi, ${ }^{c}$ and Kálmán K. Szabó ${ }^{a b}$ \\ ${ }^{a}$ University of Wuppertal, Department of Physics, Wuppertal D-42097, Germany \\ ${ }^{b}$ Jülich Supercomputing Center, Jülich D-52425, Germany \\ ${ }^{c}$ Eötvös University, Budapest 1117, Hungary \\ ${ }^{d}$ MTA-ELTE "Lendulet" Lattice Gauge Theory Research Group, Budapest, 1117, Hungary \\ ${ }^{e}$ University of Regensburg, Department of Physics, Regensburg D-93053, Germany \\ E-mail: borsanyi@uni-wuppertal.de, fodorebodri.elte.hu, \\ katz@bodri.elte.hu, simon-wolfgang.mages@ur.de, \\ nogradi@bodri.elte.hu, szaboka@general.elte.hu
}

We give a summary of our preliminary results on the finite temperature topological susceptibility $\chi$ from pure SU(3) theory. The simulations use a Symanzik improved action and a gluonic definition of the topological charge with cutoff effects at the $a^{2}$ level. We use the Wilson flow to calculate a properly renormalized topological charge and its susceptibility. Our results suggest a very strong decay of the topological susceptibility above $T_{c}$ in line with the results in literature.

The 33rd International Symposium on Lattice Field Theory

14 -18 July 2015

Kobe International Conference Center, Kobe, Japan*

\footnotetext{
* Speaker.
} 


\section{Introduction}

The standard model of particle physics is one of the best tested physical theories. Its predictions have been challenged by experimentalists for decades without finding significant discrepancies even in very fine details. There are however very significant discrepancies in the big experimental picture, as the standard model lacks e.g. a description of Dark Matter and Dark Energy as well as gravity. But it does also have some theoretical problems e.g. in explaining the values of its parameters. One of them is the strong CP problem: In principle the strong interactions can be CP violating by the introduction of a new term $\propto \theta F \tilde{F}$ which is consistent with all principles which the standard model is built on. In particular it is no problem, that it violates CP as CP is already maximally violated by the weak interaction. Experimentally one finds, that $\theta$ is zero to high precision, while theoretically it could be any angle between $(-\pi, \pi]$. Unless one accepts that $\theta$ is fine tuned to exactly zero in our universe, one needs a mechanism which dynamically sets $\theta$ to zero. A prominent candidate of these mechanisms was proposed by Peccei and Quinn [1,2] which promotes $\theta$ to a dynamical field at some scale $f_{a}$. At low energies this gives rise to a pseudo scalar particle called axion subject to an effective potential with minimum at $\theta=0$ which effectively restores $\mathrm{CP}$ symmetry at low energies. The effective potential is generated by QCD dynamics via the coupling $\propto \theta F \tilde{F}$ which leads to an effective mass of the axion given by the finite temperature topological susceptibility $\chi(T)$

$$
\chi(T)=f_{a}^{2} m_{a}(T)^{2} .
$$

If the Peccei-Quinn mechanism is realized in nature to resolve the strong CP problem, then these axion particles may also make up a substantial part of the current dark matter. As $\chi(T)$ is accessible in Lattice QCD calculations, this opens a unique possibility to use Lattice QCD to determine properties of a very well motivated Dark Matter candidate.[3, 4, 5, 6]

The topological susceptibility has been calculated on the lattice for a long time even at finite temperature.[7, 8] Very recently the interest in this topic increased again due to the new relevance of axions in experimental Dark Matter searches. This lead e.g. to pure gauge studies in a large volume and with high statistics up to $2.5 T_{c}$ [4] and the exploration of new techniques to improve the tunneling between sectors using reweighting [5]. In the end, the relevant result for the experimental axion search is the result for full QCD at physical quark masses. This is, however, very expensive and as a first step towards this final goal, we are also considering the quenched theory only. Some of the technical problems are relevant for full QCD as well, like dealing with very small topological susceptibilities at large temperature. Additionally, continuum extrapolated results at finite temperature in the quenched theory are an important crosscheck for analytical models of QCD above the phase transition.[6]

To define the topological susceptibility, one needs to define the topological charge and its density first. The topological charge $Q$ is the integral of the pseudo-scalar topological charge density $q(x)$ over some manifold $\mathscr{M}$

$$
Q=\int_{\mathscr{M}} \mathrm{d}^{4} x q(x), \quad q(x)=\frac{1}{32 \pi^{2}} \varepsilon_{\mu v \rho \sigma} \operatorname{tr}\left(F_{\mu v}(x) F_{\rho \sigma}(x)\right),
$$

with the field strength tensor $F_{\mu \nu}$ and the totally antisymmetric symbol $\varepsilon_{\mu \nu \rho \sigma}$. On a torus $\mathscr{M}=\mathbb{T}^{4}$, the topological charge is discrete and assumes integer values if the boundary condition of the gauge 
field is periodic. The sectors with different $Q$ are separated by an infinite action barrier in the continuum limit, which makes it very difficult for Monte Carlo algorithms based on performing "small" steps in field space to sample all sectors according to the probability distribution given by the Euclidean action in the path integral. This is a problem for the ergodicity requirement for the validity of the simulation and also makes it practically impossible to estimate a meaningful statistical error from the data.

The topological susceptibility is then defined as the integral of the correlator of $q(x)$

$$
\chi=\int_{\mathscr{M}} \mathrm{d}^{4} x\langle q(0) q(x)\rangle .
$$

On $\mathscr{M}=\mathbb{T}^{4}$ this integral has a natural largest distance contributing to the susceptibility given by half the lattice extent. Using global translation symmetry on $\mathscr{M}=\mathbb{T}^{4}$ one gets

$$
\chi=\frac{1}{V_{4}}\left\langle Q^{2}\right\rangle \text {. }
$$

If translation invariance is broken in any way, one cannot use Equ. 1.4 any more and one has to go back to Equ. 1.3 to calculate the physical susceptibility.[10] Using Equ. 1.4 in a setup with broken translation symmetry leads to another observable which differs from the "true" susceptibility by large finite volume errors compared to the small value of the susceptibility. Of course, in the infinite volume limit one recovers the correct result, but this can be very expensive.

Lets assume translation symmetry for the moment. Then Equ. 1.4 tells us, that in order to measure $\chi$, we have to sample also configurations with $Q \neq 0$ as otherwise the susceptibility would be zero exactly. This is very difficult to reach for simulations on $\mathscr{M}=\mathbb{T}^{4}$ close to the continuum limit as the charge becomes topologically protected from changes in the Monte Carlo evolution.

\section{Topological Susceptibility with the Wilson Flow}

Like most other observables also the topological susceptibility $\chi$ has to be properly renormalized. It is subject to both additive and multiplicative renormalization [9] with renormalization constants $Z$ and $\chi_{0}, \chi^{R}=Z \chi+\chi_{0}$. It is also known empirically, that cooling makes $Z \rightarrow 1$ and $\chi_{0} \rightarrow 0$ such that in the limit of large cooling, the renormalization becomes trivial.

The Wilson flow has the same properties as cooling with regard to the renormalization such that $\chi(t)$ at finite flow time is already renormalized.[11] But the statement for the Wilson flow has a more solid analytical footing. It has been shown additionally, that the continuum limit of the susceptibility obtained at any finite flow time $t$ is independent of $t$ and that the susceptibility obtained from the gluonic definition at finite flow time is equivalent to the fermionic definition appearing in Ward identities.[12] This means, that it is sufficient to perform a continuum limit at flow time fixed in physical units, e.g. $t=w_{0}^{2}$. The choice of $t$ only impacts the size of the lattice artefacts.

We demonstrate this by giving the flow time dependence for three different lattice spacings in Fig. 1. The dependence is very soft for larger flow time for all considered lattice spacings and the least dependence is visible at the finest lattice spacing. This indicates that the continuum limit calculated at different flow times yields the same value. For our calculations we choose $t=w_{0}^{2} \approx(0.176 \mathrm{fm})^{2}$ which is well inside the plateau region. 


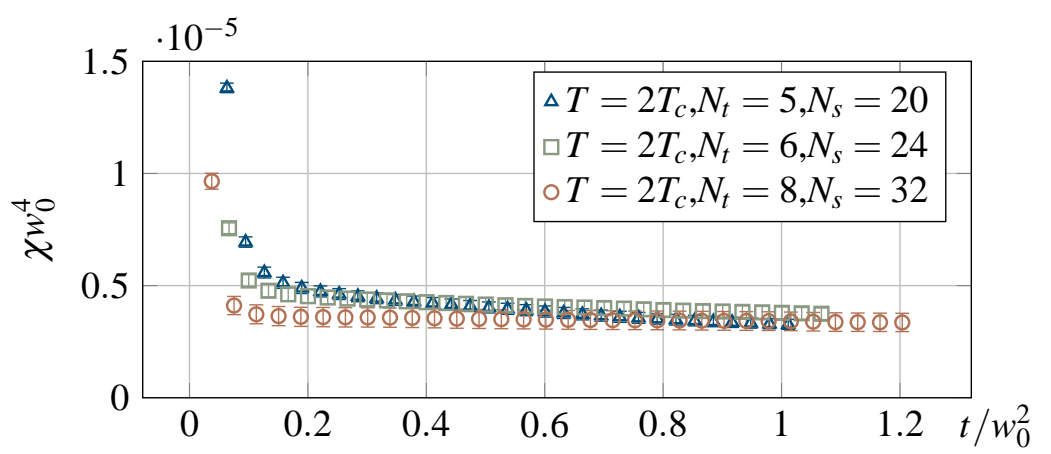

Figure 1: Flow time dependence of the topological susceptibility $\chi(t)$ for $\mathscr{M}=\mathbb{T}^{4}$ with the susceptibility calculated via the standard $Q^{2}$

For our pure gauge simulations we use a Symanzik improved gauge action with $O\left(a^{2}\right)$ errors and for measurements a gluonic topological charge density $q(x)$ calculated from the from clover field strength tensor which has also $O\left(a^{2}\right)$ errors. Each update step in the simulation consists of one heatbath and four overrelaxation steps. Our temperatures are in the range $0.1 T_{c} \leq T \leq 4.0 T_{c}$, at three different lattice spacings corresponding to $n_{t}=5,6,8$. We keep the spatial volume fixed in physical units to $L_{x, y}=2 / T_{c}$ for all temperatures. For the main runs the lattice size in $z$ direction is twice as large as the one in the other spatial directions, $L_{z}=2 L_{x, y}$, to enable a subvolume analysis (explained below in section 3).

\section{Subvolume Method}

A possible solution for the problems induced by the sampling of the discrete distribution of the topological charges $Q$ was given in Ref. [13]. The method is based on the property of the physics in any finite subvolume $V_{s u b}$ of the total volume $V_{4}$ of the lattice to behave like a finite subvolume of the theory on an infinite volume in the limit of large lattice volumes $V_{4}$. In particular, the topological charge $Q_{s u b}$ on $V_{\text {sub }}$ is not quantized on the subvolume. Therefore there are no problems sampling its distribution. The susceptibility on the subvolume is then defined as

$$
\chi_{\text {sub }}=\frac{1}{V_{\text {sub }}} Q_{\text {sub }}^{2} .
$$

To obtain the physical result, one needs to take the limit of infinite $V_{\text {sub }}$ in the end.

This strategy is physically plausible and works in cases where the susceptibility $\chi$ is large, e.g. at zero temperature in the quenched case. But for cases of small $\chi$ it does not work any more, as this definition introduces relatively large finite (sub)volume errors. These errors are independent on the size of $\chi$ itself, such that for sufficiently large temperatures or sufficiently light quark masses, one cannot simulate a large enough volume, such that the finite volume errors can be neglected.

This problem is visualized in Fig. 2. The plot shows our quenched lattice data for $T=$ $2 T_{c}, N_{t}=5$, and varying $N_{x}=N_{y}=N_{z} / 2$. We choose a subvolume of the torus as the complete lattice in all directions but the $z$ direction where we restrict the length to $L_{s u b}=L_{z} / 2$. This choice already minimizes finite volume effects as it minimizes the surface-to-volume ratio for a given large subvolume of the lattice. One can see, that using the full volume there are no $1 / L$ finite volume errors, but very strong effects for the subvolume method. 


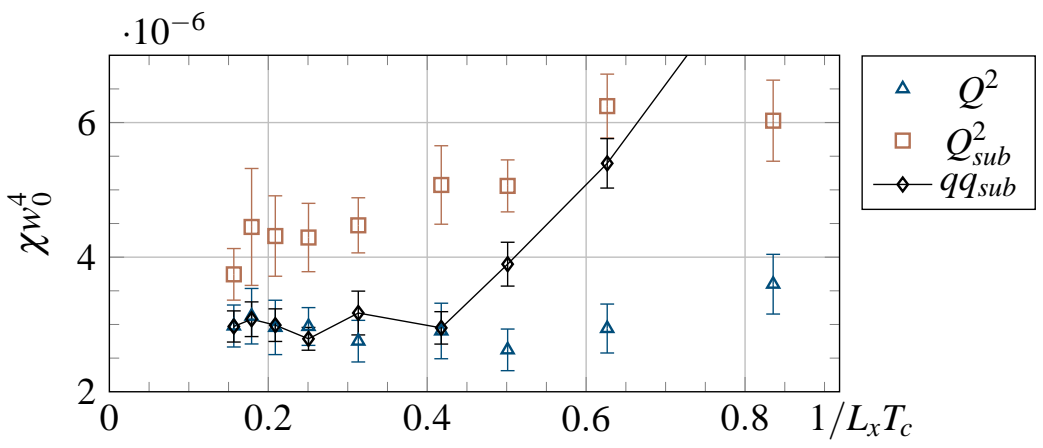

Figure 2: Finite volume errors of different definitions of the topological susceptibility at $T=2 T_{c}, N_{t}=5$, $N_{x}=N_{y}=N_{z} / 2$. $Q^{2}$ uses the full torus volume, $Q_{s u b}^{2}$ uses a subvolume of the torus which is only restricted in $z$ direction to the length $L_{s u b}$, while $q q_{s u b}$ directly evaluates the correlator definition with a maximal distance in $z$ direction of $L_{\text {sub }} / 2$.

The reason for these large finite volume errors for the subvolume susceptibility is that the step from the physical integral definition of the susceptibility Equ. 1.3 to the more common $Q^{2}$ version Equ. 1.4 required translation invariance of the manifold $\mathscr{M}$ which one integrates over. This is obviously not valid any more for the integration over a subvolume with boundary and introduces large finite volume errors. The size of the error is so large, because there are large cancellations in the integral over the correlator which has a large positive core and (up to the susceptibility equally) large negative tail. If one ignores the necessity of translation invariance and uses Equ. 3.1, one ends up with some parts of the correlator overrepresented in the susceptibility and therefore with not complete cancellations and large $1 / L$ finite volume effects.

A remedy to this problem is to introduce an infrared cutoff or a largest distance in the integral over the correlator Equ. 1.3 but to still integrate up the correlator explicitly. In this way one retains the major part of the cancellations in the integral up to exponentially suppressed contributions from the cut off exponential tail of the correlator. But by not integrating over the total volume, one should still retain some of the advantages of the subvolume method, as this integral has not the full global information on the topological charge density and is therefore not aware of the integer global topological charge. This new definition of the subvolume susceptibility is also plotted in Fig. 2. One clearly sees that the $1 / L$ finite volume errors are absent. Less clear, however, is the gain in precision which one expects from using only information on subvolumes compared to the version using the total volume, but there is also a small gain visible.

\section{Finite Temperature Results}

For the simulations in the large range of temperatures of our main production runs we see a consistent behaviour of the finite volume effects as given in 3. For small temperatures at high susceptibilities the finite volume errors are not relevant. But for small susceptibilities like those at very high temperatures one cannot use the subvolume susceptibility from Equ. 3.1. The subvolume correlator definition from Equ. 1.3 restricted to a finite subvolume gives results compatible to the total volume definition Equ. 1.4. But as also the errors for large temperatures are consistent, in the remainder we will discuss only our results from the standard definition on the total volume. 


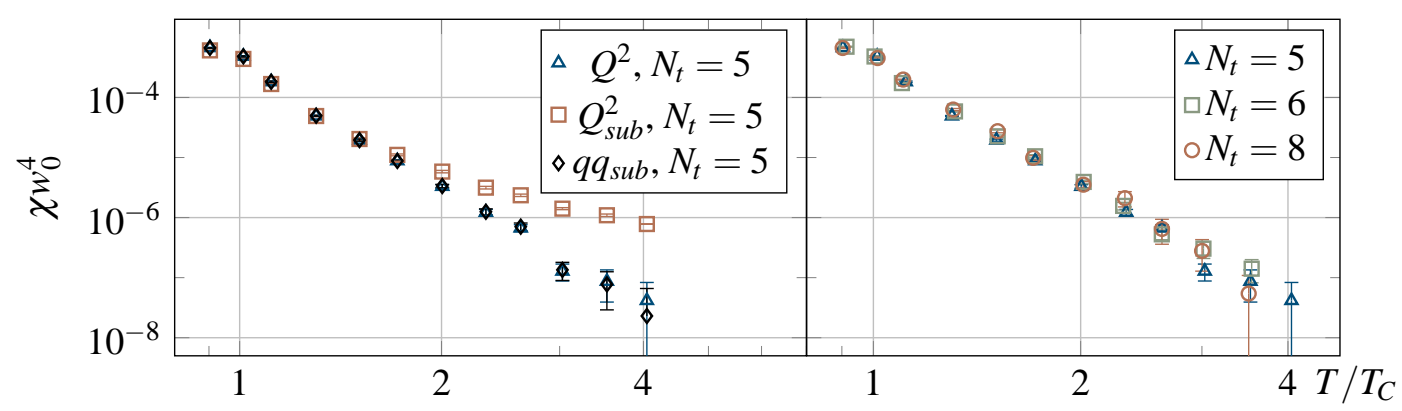

Figure 3: Left: Demonstration of the finite volume effects in the subvolume definition of the topological susceptibility $Q_{\text {sub }}^{2}$ compared to the full volume susceptibility $Q^{2}$ and the subvolume correlator definition $q q_{s u b}$ for our $N_{t}=5$ ensembles. Right: Lattice spacing dependence of the topological susceptibility from the full volume $Q^{2}$ for our three lattice spacings at $N_{t}=5,6,8$.

In order to produce physical results we also have to perform a continuum extrapolation. Fig. 3 gives our results for $N_{t}=5,6,8$. We see that the lattice spacing dependence is mild. As we have $O\left(a^{2}\right)$ improvement on the action and on the observables this behaviour is reasonable.

In Fig. 4 we compare our $N_{t}=5$ results with results given in literature. For this ensemble we also performed a fit to the standard ansatz of the topological susceptibility decaying like a power of the temperature. While the results presented here are preliminary, we see a significant discrepancy to the high statistics result of [4] while we are quite consistent with the other results in literature.

\section{Discussion and Outlook}

In this contribution we have discussed some technical advances in the quest to get a reliable Lattice QCD result for the susceptibility at large finite temperature like it is necessary for axion phenomenology. All of our results are for the quenched case. We have used a gluonic definition for the topological charge density at finite Wilson flow time and have extended the covered temperature range to $4 T_{c}$. We have also demonstrated that the subvolume method does not work for small susceptibilities as it has large $1 / L$ finite volume effects. We have found the reason for this pathological behaviour and we have presented a cure for it.

The next steps are to generate more data for the fine lattice spacings to be able to perform a

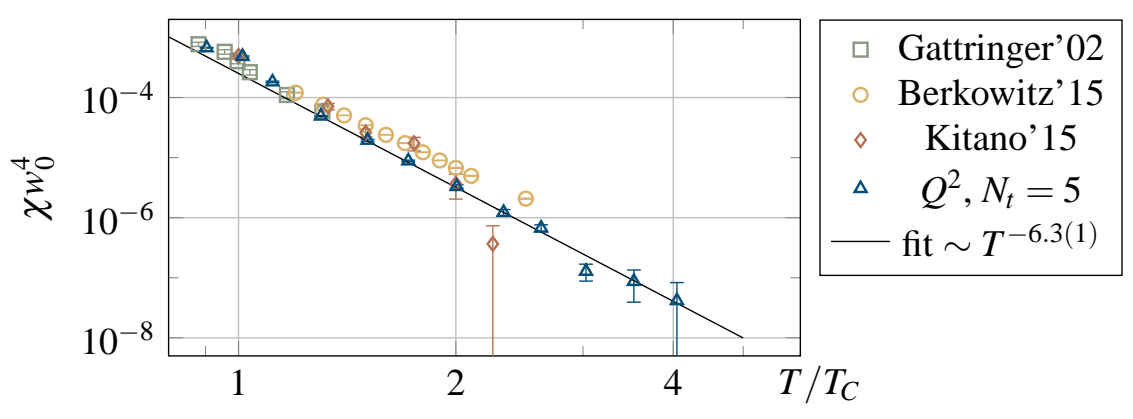

Figure 4: Comparison of our preliminary $N_{t}=5$ results with results in literature. 
controlled continuum extrapolation of the quenched result. ${ }^{1}$ Also we plan to tune the subvolume method in order to increase the gain in statistical accuracy to make it a helpful tool in the difficult task to determine the susceptibility even for the unquenched case, which has a much smaller topological susceptibility. In addition it is more difficult to reach high temperatures in the $\mathrm{GeV}$ region, since $T_{c}$ is smaller than in the quenched case (c.f. [14],[15],[16]), thus larger $T / T_{c}$ values are needed.

\section{Acknowledgements}

This work was supported by OTKA under grant OTKA-NF-104034. The simulations were performed on the QPACE machines funded by the DFG grant SFB/TR55.

\section{References}

[1] R. D. Peccei and H. R. Quinn, Phys. Rev. Lett. 38 (1977) 1440. doi:10.1103/PhysRevLett.38.1440

[2] R. D. Peccei and H. R. Quinn, Phys. Rev. D 16 (1977) 1791. doi:10.1103/PhysRevD.16.1791

[3] O. Wantz and E. P. S. Shellard, Phys. Rev. D 82 (2010) 123508 doi:10.1103/PhysRevD.82.123508 [arXiv:0910.1066 [astro-ph.CO]].

[4] E. Berkowitz, M. I. Buchoff and E. Rinaldi, arXiv:1505.07455 [hep-ph].

[5] R. Kitano and N. Yamada, arXiv:1506.00370 [hep-ph].

[6] S. Borsanyi et al., Phys. Lett. B 752 (2016) 175 doi:10.1016/j.physletb.2015.11.020 [arXiv:1508.06917 [hep-lat]].

[7] B. Alles, M. D’Elia and A. Di Giacomo, Nucl. Phys. B 494 (1997) 281 [Nucl. Phys. B 679 (2004) 397] [hep-lat/9605013].

[8] C. Gattringer, R. Hoffmann and S. Schaefer, Phys. Lett. B 535 (2002) 358 doi:10.1016/S0370-2693(02)01757-4 [hep-lat/0203013].

[9] B. Alles, M. D'Elia, A. Di Giacomo and R. Kirchner, Phys. Rev. D 58 (1998) 114506 [hep-lat/9711026].

[10] S. Mages, B. C. Toth, S. Borsanyi, Z. Fodor, S. Katz and K. K. Szabo, arXiv:1512.06804 [hep-lat].

[11] M. Lüscher, JHEP 1008 (2010) 071 [JHEP 1403 (2014) 092] doi:10.1007/JHEP08(2010)071, 10.1007/JHEP03(2014)092 [arXiv:1006.4518 [hep-lat]].

[12] M. Cé, C. Consonni, G. P. Engel and L. Giusti, Phys. Rev. D 92, no. 7, 074502 (2015) doi:10.1103/PhysRevD.92.074502 [arXiv:1506.06052 [hep-lat]].

[13] R. C. Brower et al. [LSD Collaboration], Phys. Rev. D 90 (2014) 1, 014503 [arXiv:1403.2761 [hep-lat]].

[14] Y. Aoki, Z. Fodor, S. D. Katz and K. K. Szabo, Phys. Lett. B 643 (2006) 46 doi:10.1016/j.physletb.2006.10.021 [hep-lat/0609068].

[15] Y. Aoki, S. Borsanyi, S. Durr, Z. Fodor, S. D. Katz, S. Krieg and K. K. Szabo, JHEP 0906 (2009) 088 doi:10.1088/1126-6708/2009/06/088 [arXiv:0903.4155 [hep-lat]].

[16] S. Borsanyi et al. [Wuppertal-Budapest Collaboration], JHEP 1009 (2010) 073 doi:10.1007/JHEP09(2010)073 [arXiv:1005.3508 [hep-lat]].

\footnotetext{
${ }^{1}$ After the conference this has been done and published in [6].
} 